\title{
Ketamine alone or combined with midazolam or dexmedetomidine does not affect anxiety-like behaviours and memory in adult Wistar rats
}

\author{
Ana Magalhães ${ }^{1,2,3}$, Ana Valentim ${ }^{1,3,4}$, Carlos Venâncio ${ }^{5,6}$, \\ Mariana Pereira7, Pedro Melo ${ }^{7}$, Teresa Summavielle ${ }^{1,2,7}$ and \\ Luis Antunes $1,4,5,6$
}

\begin{abstract}
Ketamine administration has been associated with controversial behavioural impairments and psychotic episodes. Even though ketamine alone and in combination with midazolam or dexmedetomidine are frequently used in laboratory animals, the side-effects of such protocols are not well known. Therefore, our aim was to evaluate the effects of ketamine alone and in combination with midazolam or dexmedetomidine on emotional reactivity, as well as the effects on learning and memory in adult rats at least $48 \mathrm{~h}$ after anaesthesia. The evaluation of the potential influence of $100 \mathrm{mg} / \mathrm{kg}$ ketamine administered alone and in combination with midazolam $(5 \mathrm{mg} / \mathrm{kg})$, or dexmedetomidine $(0.25 \mathrm{mg} / \mathrm{kg}$ ) on spatial learning and recognition memory was studied in adult Wistar rats using the radial maze as well as object recognition and location tests. The influence of these combinations on emotional reactivity was investigated using the new exploration test and the elevated plus maze. Results showed that ketamine alone or in combination with midazolam or dexmedetomidine affected neither spatial and recognition memory, nor emotional reactivity. These results reinforce the safe clinical use of ketamine and its combinations in rats in a research context since the administration of these anaesthetic combinations did not produce significant changes with regard to spatial and recognition memory or emotional reactivity. Furthermore, these results indicate that the quality of scientific data produced in adult rat neurobehavioural research is not jeopardized by the use of these anaesthetic protocols.
\end{abstract}

\section{Keywords}

anaesthetic combinations, ketamine, midazolam, memory, Wistar rat, anxiety-like behaviours

Anaesthesia is used during surgery and other interventions to control pain, anxiety and consciousness. In veterinary practice, anaesthesia is also essential in immobilizing animals for clinical examination., ${ }^{1,2}$ Likewise, anaesthesia is required for several procedures in research involving laboratory animals, especially in neurobiological research.

All experiments using laboratory animals should be based on the 'three R principle': refinement, replacement and reduction. ${ }^{3}$ Refinement of anaesthesia should act as an important parameter in consideration of, not only the
${ }^{1}$ Institute for Research Innovation in Health, Rua Júlio Amaral de Carvalho, Porto, Portugal

${ }^{2}$ Addiction Biology, Institute for Molecular and Cellular Biology, University of Porto, Rua do Campo Alegre, Porto, Portugal

${ }^{3}$ Behavioral Science Department, Institute of Biomedical Sciences Abel Salazar, University of Porto, Rua de Jorge Viterbo Ferreira, Porto, Portugal

${ }^{4}$ Laboratory of Animal Science, Institute for Molecular and Cellular Biology, University of Porto, Rua do Campo Alegre, Porto, Portugal ${ }^{5}$ School of Agrarian and Veterinary Sciences, University of Trás-osMontes and Alto Douro, Quinta dos Prados, Vila Real, Portugal

${ }^{6}$ Centre for the Research and Technology of Agro-Environmetal and Biological Sciences, CITAB, University of Trás-os-Montes and Alto Douro, Quinta dos Prados, Vila Real, Portugal

${ }^{7}$ Functional Sciences Department, High Institute for Allied Health Technologies, Polytechnic Institute of Porto, Vila Nova de Gaia, Portugal 
promotion of animal welfare, but also the avoidance of anaesthetics that may interfere with experimental results.

In research using laboratory animals the N-methyl-Daspartate (NMDA) antagonist ketamine is probably the most frequently used anaesthetic. In humans, ketamine is described as a dissociative anaesthetic and is still used worldwide in clinical settings ${ }^{4}$ and in emergencies. ${ }^{5}$

The use of ketamine is limited because of its psychotic side-effects, ${ }^{6}$ which can be overcome by finding a balanced anaesthesia. This technique allows for safe anaesthesia by decreasing the doses required for each individual drug and still achieving a clinical effect. Therefore, ketamine is frequently used in combination with midazolam or dexmedetomidine. ${ }^{7,8}$ Besides providing anterograde amnesia, the benzodiazepine midazolam is also used as a sedative and anxiolytic compound. ${ }^{9}$ Dexmedetomidine is a recent $\alpha 2$-adrenergic agonist that also induces sedation, anxiolysis, and analgesia. ${ }^{10}$

Acute and repeated sub-anaesthetic doses of ketamine have been reported to impair cognition in humans ${ }^{11}$ and rodents. ${ }^{12,13}$ However, acute ketamine administration has also been shown to attenuate post-operative cognitive dysfunction. ${ }^{14}$ Regardless of all the evidence, the literature is nonetheless scarce regarding the effects of ketamine in combination with dexmedetomidine or with midazolam on long-term memory. Studies in humans have reported that these combinations produce anterograde amnesia and anxiolysis, and reduce the side-effects related with ketamine. ${ }^{15,16}$ The ketamine/midazolam combination also causes transient and dose-dependent memory impairment. ${ }^{15,17}$ Our group has recently reported that this anaesthetic combination did not disrupt the learning process of mice during a spatial task, ${ }^{18,19}$ and that ketamine combined with medetomidine also did not affect their performance in the T-maze test. ${ }^{20}$ Thus, it has become imperative to evaluate the cognitive functions of rats after being subjected to these anaesthetic protocols.

Therefore, in the present study, we evaluated the effects of the ketamine alone and in combination with midazolam or dexmedetomidine on emotional reactivity, and on learning and memory in rats - one of the most frequently used animal models in neuroscience research. This study attempts to replicate what happens in research and contributes to the refinement of anaesthesia in laboratory animals by evaluating this combination when rats are anaesthetized and used in neurobehavioural research.

\section{Materials and methods}

\section{Animals}

Eighty-two male Wistar rats (from the colony of the Institute of Molecular and Cell Biology, University of Porto, Portugal) aged 10-12 weeks were used in the study. Rats were housed in pairs in a controlled environment $\left(20 \pm 2^{\circ} \mathrm{C}, 45-55 \%\right.$ humidity $)$ with a $12 \mathrm{~h}$ light/dark cycle. Food and water were supplied ad libitum. All behavioural experiments were performed during the dark phase, except for the elevated plus maze (EPM) test. All procedures were carried out under personal and project licences approved by the national authority for animal protection, 'Direção Geral de Alimentação e Veterinária' (Lisbon, Portugal), and were performed in accordance with the European Directive 2010/63/EU on the protection of animals used for scientific purposes.

\section{Anaesthesia}

Ketamine (Imalgene 1000, Merial, Lisbon, Portugal; $100 \mathrm{mg} / \mathrm{mL}$ ), midazolam (B Braun Medical, Barcelona, Spain; $5 \mathrm{mg} / \mathrm{mL}$ ), and dexmedetomidine (Dexdomitor, Esteve Farma, Lda, Carnaxide, Portugal, $0.5 \mathrm{mg} / \mathrm{mL}$ ) were dissolved in a sterile saline solution (Braun Vet, Queluz de Baixo, Portugal) before intraperitoneal injection at $1 \mathrm{~mL} / \mathrm{kg}$ of body weight. Rats were randomly distributed into four groups: CONTROL (saline solution, $n=18), 100 \mathrm{mg} / \mathrm{kg}$ ketamine (KET, $n=19), 100 \mathrm{mg} / \mathrm{kg}$ ketamine and $5 \mathrm{mg} / \mathrm{kg}$ midazolam (KET/MID, $n=19$ ), and $100 \mathrm{mg} / \mathrm{kg}$ ketamine and $0.25 \mathrm{mg} / \mathrm{kg}$ dexmedetomidine (KET/DEX, $n=19$ ); the combination of drugs was administered in a single injection. In addition to the groups previously referred to, and in order to evaluate emotional reactivity using the EPM test and to avoid prolonged periods of anaesthesia, KET/DEX $(100 \mathrm{mg} / \mathrm{kg}$ and $0.25 \mathrm{mg} / \mathrm{kg}$ ) anaesthesia was reverted using atipamezole (Antisedan, Pfizer, Oeiras, Portugal; $5 \mathrm{mg} / \mathrm{mL}$ ) one hour after loss of righting reflex (KET/DEX+A).

After anaesthetic administration, the animals were isolated until righting reflex was lost. Subsequently, animals were placed on a homeothermic blanket, connected to a rectal thermal probe (50-7061-F; Harvard Apparatus Ltd, Kent, UK) to maintain a body temperature at $37 \pm 1{ }^{\circ} \mathrm{C}$. During anaesthesia, rats received oxygen through a facemask, and their eyes were kept humid with sterile saline solution. Oxygen saturation was maintained between 90 and $99 \%$ using a pulse oximetry (Vitalstore Multimonitor base unit; Vetronics, Devon, UK) with a sensor placed in the hindlimb of each animal. Respiratory and pulse rates were measured using the same equipment every $5 \mathrm{~min}$. Time to lose and time to gain the righting reflex were also recorded.

\section{Experiment 1: Evaluation of the potential influence of ketamine alone and combined with midazolam or dexmedetomidine on spatial learning and recognition memory}

The cognitive status was assessed in the eight-radial maze and in the object recognition and location 
$(\mathrm{OR} / \mathrm{L})$ tests. Forty-eight animals were randomly distributed between the radial maze and the OR/L tests. Six animals were allocated to each of the experimental groups (CONTROL, KET, KET/MID and KET/ DEX). The radial maze training and the habituation to $\mathrm{OR} / \mathrm{L}$ started $48 \mathrm{~h}$ after the anaesthesia protocols.

Radial maze test. Working and reference memories were assessed simultaneously through a fixed position of reward task, in which three of the arms were baited and their positions were fixed throughout the training trials. The entry into the never-baited arm was considered to be a reference memory (RM) error. Within a training session, the re-entry into one of the arms was considered a working memory (WM) error. ${ }^{21}$ The response latency was defined as the total duration of the experiment and the total number of visited arms.

The radial maze was made of black Plexiglas (Panlab, SLU, Barcelona, Spain) and elevated $50 \mathrm{~cm}$ from the ground. It consisted of a central area with eight sliding doors giving access to eight equally-sized arms. For reinforcement, three sugar pellets (Bio-Serv, Frenchtown, NJ, USA) were placed in the distal end of each arm. External-maze cues were added to aid navigation. The amount of time a rat took to complete a trial was recorded. During experiments, animals had restricted access to food, and were being fed only following testing. The body weight loss of animals was kept below $10 \%$.

Habituation began five days before the training period. On three consecutive days, sugar pellets were distributed and rats were allowed to individually explore the maze for $5 \mathrm{~min}$ or until the pellets were consumed. Twenty-four hours after the habituation period, animals were anaesthetized.

Training started $48 \mathrm{~h}$ after anaesthesia, with daily sessions (five trials per session) over a nine-day period. At the beginning of each trial, rats were placed in the centre of the maze and always facing the same arm, and were allowed to explore the maze for 5 min or until all the pellets were consumed. An arm entry was confirmed when all four paws were inside the arm of the maze. After each session animals were returned to their home cage and were fed. Between sessions, the maze was cleansed with absorbing paper and a $30 \%$ ethanol solution to minimize the olfactory cues. In addition to minimizing these cues, maze rotation was made between trials.

Object recognition/location test. The OR test was performed as described in a previous work. ${ }^{13}$ Briefly, the test apparatus consisted of an open box and the objects used were made of plastic, glass or metal in three different shapes: cubes, pyramids and cylinders. The test consisted of three phases.
Habituation began $48 \mathrm{~h}$ after anaesthesia and rats were allowed to explore the apparatus for $10 \mathrm{~min}$, over five consecutive days. In all sessions, the arena contained a single object (a familiar object) placed in the centre. The following day, sample phase OR1 started by placing each rat in the apparatus with two identical objects (familiar) for $3 \mathrm{~min}$ and then the rat was removed to its home cage. The choice phases were performed after a $15 \mathrm{~min}$ (OR2) and $24 \mathrm{~h}$ (OR3) delay. In this phase the apparatus contained a novel object and a copy of the previously seen familiar object, and animals were allowed to explore the objects for $3 \mathrm{~min}$. The time spent exploring the novel object served as the measure of recognition memory for the familiar object. Exploration was defined as follows: rat touched the object with its nose or the rat's nose was directed toward the object at a distance shorter than $2 \mathrm{~cm}$. Circling or sitting on the object was not considered to be exploratory behaviours. The index of discrimination was calculated as the difference between exploration time of the novel and familiar objects. ${ }^{22}$

OL testing was performed $24 \mathrm{~h}$ after the OR test. The sample phase of the OL1 test was exactly the same as for the recognition test. After a retention interval of $15 \mathrm{~min}$, the rat was returned to the apparatus for 3 min with both objects, one of which was placed in the previously used location, and the other placed near the adjacent corner, a new location - choice phase (OL2). This task assessed the ability to discriminate the novelty of the OL. ${ }^{22}$ Data from the OR/L tests were analysed using the Observer 10 software (Noldus Information Technology, Wageningen, The Netherlands).

\section{Experiment 2: Evaluation of the potential influence of ketamine alone and combined with midazolam or dexmedetomidine on emotional reactivity}

The emotional behaviours were assessed in the new object exploration (NOE) test and the EPM test $48 \mathrm{~h}$ after anaesthesia. Thirty-four animals were randomly distributed to each of the experimental groups (KET, $\mathrm{KET} / \mathrm{MID}, \mathrm{KET} / \mathrm{DEX}$ and KET/DEX+A, $n=7$, except CONTROL with $n=6$ ) in the EPM test. The 24 animals used in the NOE were the same as those used during the first day of habituation to OR/L. Tests began $48 \mathrm{~h}$ after anaesthesia.

All behaviours were video-recorded with a camera placed above the apparatus and saved to a computer by the multi-camera vigilance GeoVision system (GV-800/ 8; GeoVision Inc, Taipei, Taiwan). Behaviours were analysed using the Observer 10 software. 
New object exploration test. In this test, each rat was placed in the open box facing away from the test object, which was placed in the centre of the open box, and allowed to explore the environment for $10 \mathrm{~min}$. The pattern of space occupation, frequency and duration of behaviours towards the object were recorded.

Elevated plus maze test. The EPM was made of dark grey plastic and positioned $50 \mathrm{~cm}$ above the floor. The maze consisted of four arms arranged in the shape of a cross, two arms had surrounding walls (closed arms) and the other two opposing arms had no walls (open arms). A test session was started by placing each rat on the central platform, facing an open arm. Over a period of $5 \mathrm{~min}$, each rat's exploration was video-recorded. The maze was cleansed after each training session. The number of open and closed arm entries, and the time spent in the different compartments of the maze (central platform, open and closed arms) were scored. As mentioned previously, the KET/DEX+A group was added to this test in order to clarify the potential influence of anaesthesia duration on anxiety.

\section{Statistical analysis}

The number of animals used per group was based on previous work ${ }^{23,24}$ with a power of 0.8 and $\alpha=0.05$. Parametric data were analysed with one-way analysis of variance (ANOVA) and the paired Student's $t$-test $(\mathrm{OR} / \mathrm{L}$ data for comparisons between groups and for differences within groups, respectively), and ANOVA for repeated measures with Bonferroni post hoc tests (random access memory (RAM) data with anaesthesia groups as a between-subject factor and day of testing as a within-subject factor). These were expressed as mean + SEM. Non-parametric data, such as anaesthetic parameters and NOE data, were analysed with the Kruskal-Wallis test followed by the MannWhitney $U$-test and expressed as median and $95 \%$ confidence intervals. A value of $P \leq 0.05$ was considered to be statistically significant.

All results were analysed using Prism 6 for data management and SPSS 16.0 (Apache Software Foundation, Forest Hill, MD, USA) for statistical analysis.

\section{Results}

\section{Anaesthesia}

Both the loss of consciousness and the anaesthesia induction time were similar between groups. The KET/MID group had a significantly higher heart rate compared with the KET/DEX and KET/DEX+A groups at several time points $(5,10,25,30$ and
$45 \mathrm{~min} ; P \leq 0.043$ ), and the KET group had a higher heart rate than the $\mathrm{KET} / \mathrm{DEX}+\mathrm{A}$ group at $10 \mathrm{~min}$ $(P=0.039)$; no differences were detected between groups regarding the respiratory rate (Table 1). Anaesthesia induction times were significantly different between groups $(\mathrm{H}(2)=44.53 ; P \leq 0.014)$. The KET group took less time to recover their righting reflex (31.2 [24.6-37.9] $\mathrm{min}$ ), followed by the KET/MID $(60.1[50.0-70.3] \mathrm{min})$ and the KET/DEX (228.3 [198.1-258.4] min) groups. KET/DEX+A anaesthesia duration was not evaluated since the anaesthesia was reverted after one hour.

\section{Behavioural tests}

Experiment 1. Animals ranging from 205 to $315 \mathrm{~g}$ were used in all behavioural tests; no body weight loss higher than $10 \%$ was observed.

The analysis of the radial arm maze data revealed a main effect of day for response latency $\left[F_{(1,22)}=30.995\right.$, $P<0.001]$, for working memory $\left[F_{(1,22)}=12.01\right.$, $P<0.001]$ and reference memory $\left[F_{(1,22)}=27.551\right.$, $P<0.001]$ errors. However, there was no main effect of treatment $(P>0.05)$ or treatment/day interactions $(P>0.05)$. In all groups the number of reference and working memory errors decreased across learning sessions (Figure 1a and b).

After the habituation phase (Figure 2a), data from OR/L showed no differences between groups. All subjects recognized the novelty of the object, as indicated by the significant increase in exploration of the new object (i.e. robust recognition memory) during the short delay of $15 \mathrm{~min}$ (OR2; CONTROL $t=-6.529$, $P<0.001 ; \quad$ KET $t=-7.260, \quad P<0.001 ; \quad$ KET $/$ MID $t=-6.422, \quad P<0.001 ; \quad \mathrm{KET} / \mathrm{DEX} \quad t=-5.053$, $P<0.01$ ), and during the long-term delay of $24 \mathrm{~h}$ (OR3; CONTROL $t=-10.736, \quad P<0.001 ; \quad$ KET $t=-5.33, P<0.01 ; \mathrm{KET} / \mathrm{MID} t=-6.024, P<0.01$; KET/DEX $t=-3.532, P<0.05)$. Furthermore, no differences between groups were found in the discrimination index (proportion of exploration time) in OR2 and OR3 (Figure 2b and c). Rats from all experimental groups also recognized the object displacement (new location), as indicated by the preferential exploration compared with the object in the familiar location (OL2; CONTROL $t=-10.253, P<0.001$; KET $t=-9.067$, $P<0.001 ; \mathrm{KET} / \mathrm{MID} t=-7.832, \quad P<0.001 ; \mathrm{KET} /$ DEX $t=-8.543, P<0.001)$. No differences between groups were found in the discrimination index in OL2 (Figure 2d).

Experiment 2. In the NOE test, the Kruskal-Wallis and Mann-Whitney results revealed that KET/DEX rats spent more time in the corners (173.0 [49.7-297.3] s) when compared with the CONTROL rats 
Table 1. Physiological parameters during $60 \mathrm{~min}$ of anaesthesia.

\begin{tabular}{|c|c|c|c|c|c|}
\hline $\begin{array}{l}\text { Time } \\
\text { (min) }\end{array}$ & $\begin{array}{l}\text { Physiological } \\
\text { parameters }\end{array}$ & KET & KET/MID & KET/DEX & $\mathrm{KET} / \mathrm{DEX}+\mathrm{A}$ \\
\hline \multirow[t]{2}{*}{5} & Heart rate (bpm) & 360.7 (307.2-414.3) & $403.0(334.5-471.5)$ & 248.8 (205.9-291.7)* & $248.4(210.2-286.7)^{*}$ \\
\hline & Respiratory rate (brpm) & $113.0(49.5-176.5)$ & $96.0(0-197.7)$ & $64.5(49.8-79.2)$ & $58.3(44.7-72.0)$ \\
\hline \multirow[t]{2}{*}{10} & Heart rate (bpm) & $393.2(294.9-491.6)^{* *}$ & $369.1(336.8-455.5)$ & $229.7(166.0-293.4)^{*}$ & $241.8(215.4-268.3)^{*}$ \\
\hline & Respiratory rate (brpm) & $91.3(47.6-135.1)$ & 95.2 (50.7-139.8) & $71.7(40.2-103.3)$ & $72.3(48.4-96.3)$ \\
\hline \multirow[t]{2}{*}{15} & Heart rate (bpm) & $304.0(225.1-382.8)$ & $294.8(124.3-465.3)$ & 253.5 (231.7-275.3) & $244.4(221.6-267.2)$ \\
\hline & Respiratory rate (brpm) & - & $67.0(0-321.1)$ & $61.5(52.4-70.6)$ & $53.0(29.8-76.2)$ \\
\hline \multirow[t]{2}{*}{20} & Heart rate $(\mathrm{bpm})$ & $300.5(0-1272.5)$ & $261.3(198.2-324.5)$ & $274.4(220.9-273.9)$ & $229.4(207.3-251.5)$ \\
\hline & Respiratory rate (brpm) & - & $83.3(-17.9-184.6)$ & $70.7(52.5-88.8)$ & $55.7(26.4-85.1)$ \\
\hline \multirow[t]{2}{*}{25} & Heart rate (bpm) & - & $295.7(161.6-429.8)$ & $230.0(208.9-251.1)^{*}$ & $218.9(194.4-243.3)^{*}$ \\
\hline & Respiratory rate (brpm) & - & - & $54.7(39.8-69.6)$ & $60.0(17.5-102.4)$ \\
\hline \multirow[t]{2}{*}{30} & Heart rate (bpm) & - & $325.7(278.9-372.6)$ & $224.7(199.7-249.7)^{*}$ & $204.9(165.3-244.4)^{*}$ \\
\hline & Respiratory rate (brpm) & - & $95.0(36.5-153.5)$ & $68.6(23.4-113.8)$ & $63.2(41.3-85.2)$ \\
\hline \multirow[t]{2}{*}{35} & Heart rate $(\mathrm{bpm})$ & - & $285.7(93.2-478.3)$ & $182.0(86.1-277.9)$ & $217.0(198.8-235.2)$ \\
\hline & Respiratory rate (brpm) & - & $127.3(105.9-148.7)$ & $66.7(40.3-93.0)$ & $69.0(37.1-100.8)$ \\
\hline \multirow[t]{2}{*}{40} & Heart rate $(\mathrm{bpm})$ & - & $334.5(137.6-531.4)$ & $213.8(185.6-242.0)$ & 214.7 (193.5-235.9) \\
\hline & Respiratory rate (brpm) & - & - & $64.0(39.2-88.8)$ & $67.7(41.4-94.1)$ \\
\hline \multirow[t]{2}{*}{45} & Heart rate $(\mathrm{bpm})$ & - & $348.0(-325.4-1021.4)$ & $171.9(98.5-245.2)^{*}$ & 218.5 (199.1-237.9)* \\
\hline & & - & - & 70.0 (57.1-82.9) & 78.4 (39.5-117.3) \\
\hline \multirow[t]{2}{*}{50} & Heart rate (bpm) & - & $402.0(58.9-745.1)$ & 188.0 (142.2-233.8) & $208.0(177.6-238.4)$ \\
\hline & Respiratory rate (brpm) & - & - & $60.4(46.3-74.5)$ & $59.0(10.1-107.9)$ \\
\hline \multirow[t]{2}{*}{55} & Heart rate (bpm) & - & $334.5(74.0-594.9)$ & $200.3(176.5-224.0)$ & 213.4 (191.9-234.9) \\
\hline & Respiratory rate (brpm) & - & - & $60.0(53.7-66.3)$ & $79.0(31.0-127.0)$ \\
\hline \multirow[t]{2}{*}{60} & Heart rate $(\mathrm{bpm})$ & - & - & $201.1(175.6-226.7)$ & $205.8(177.5-234.1)$ \\
\hline & Respiratory rate (brpm) & - & - & 73.5 (59.7-87.3) & $69.0(0-323.1)$ \\
\hline
\end{tabular}

$\mathrm{KET}: 100 \mathrm{mg} / \mathrm{kg}$ of ketamine; KET/MID: $100 \mathrm{mg} / \mathrm{kg}$ of ketamine and $5 \mathrm{mg} / \mathrm{kg}$ of midazolam in a single injection; KET/DEX: $100 \mathrm{mg} / \mathrm{kg}$ of ketamine and $0.25 \mathrm{mg} / \mathrm{kg}$ of dexmedetomidine in a single injection; KET/DEX+A: $100 \mathrm{mg} / \mathrm{kg}$ of ketamine and $0.25 \mathrm{mg} / \mathrm{kg}$ of dexmedetomidine in a single injection, and $5 \mathrm{mg} / \mathrm{kg}$ of atipamezole one hour post-anaesthesia; bpm: beats per minute; brpm: breaths per minute. Data are shown as median (95\% confidence interval). ${ }^{*} P \leq 0.043$ for comparison with KET/MID; ${ }^{* *} P=0.039$ for comparison with $\mathrm{KET} /$ $\mathrm{DEX}+\mathrm{A}$. When no value is exhibited this implies that the animals were too superficial, and the equipment could not measure the variable.

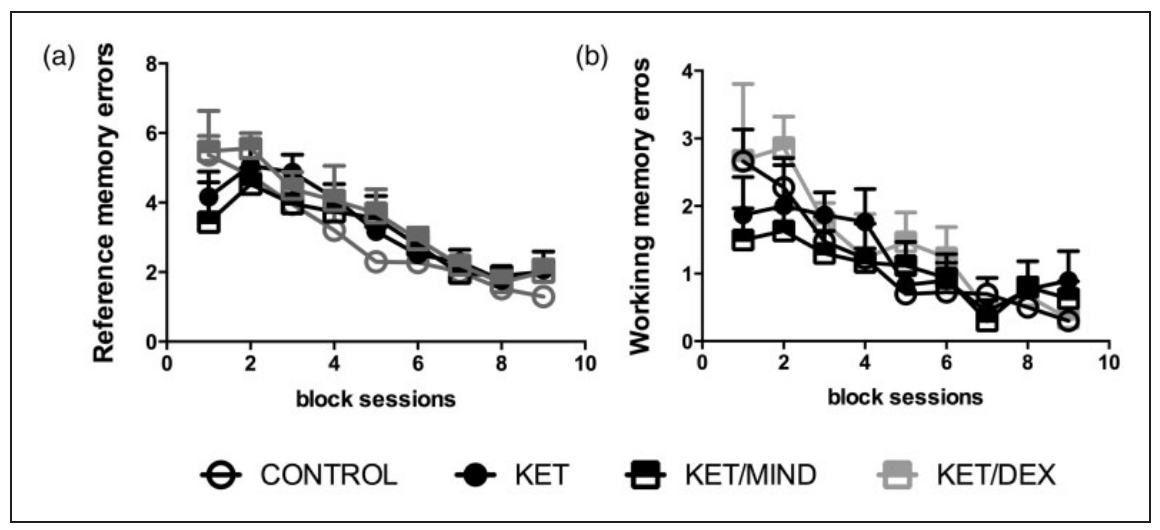

Figure 1. Reference (a) and working (b) memory errors tested in radial maze in rats exposed to ketamine alone, or combined with midazolam or dexmedetomidine. Results are presented as means + SEM $(n=6)$ for groups: saline solution (CONTROL); $100 \mathrm{mg} / \mathrm{kg}$ ketamine (KET); $100 \mathrm{mg} / \mathrm{kg}$ ketamine and $5 \mathrm{mg} / \mathrm{kg}$ midazolam (KET/MID); and $100 \mathrm{mg} / \mathrm{kg} \mathrm{ketamine}$ and $0.25 \mathrm{mg} \mathrm{kg}$ dexmedetomidine (KET/DEX); block session was calculated as average measured on five trials. No differences between groups were observed. 


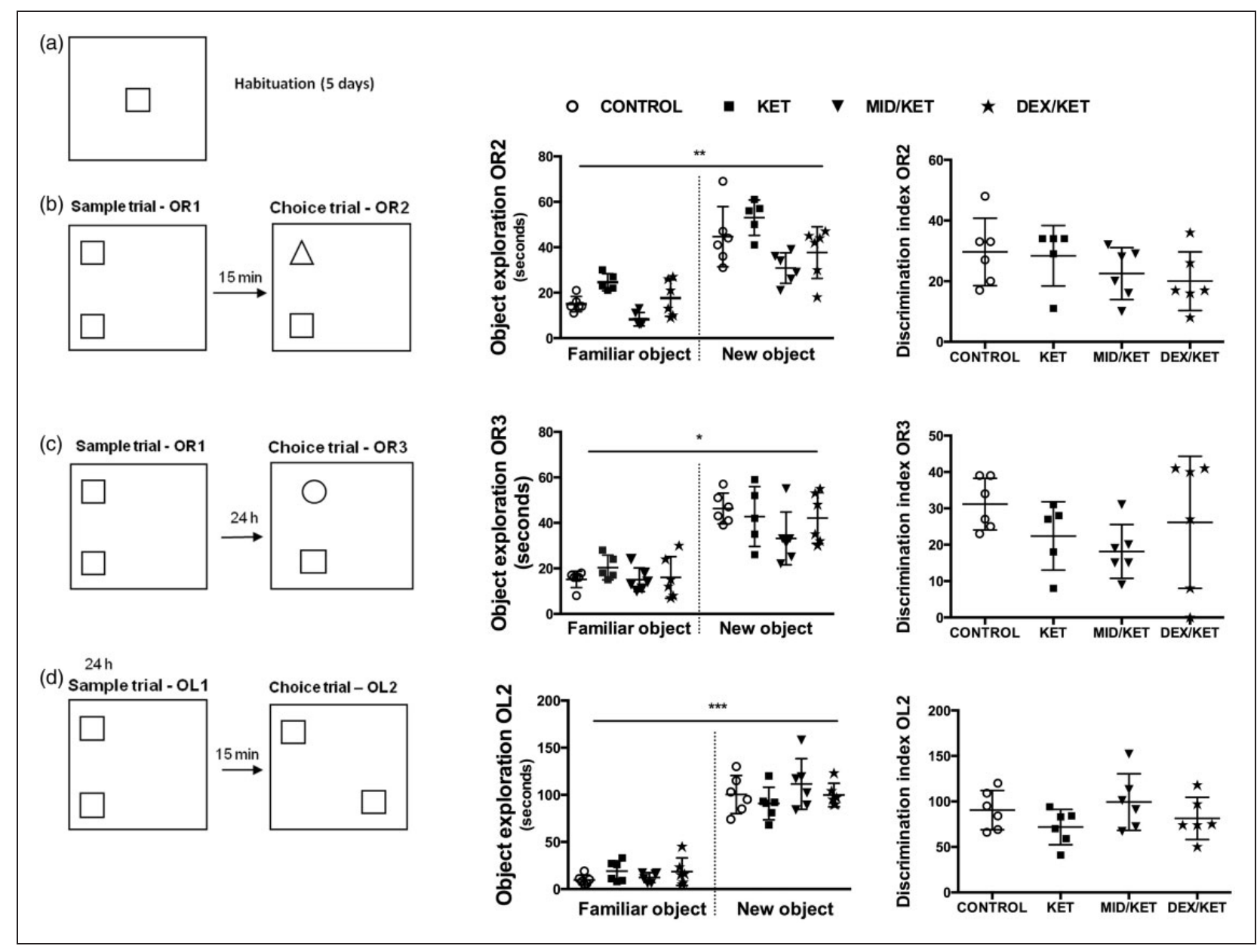

Figure 2. Object recognition (OR) and object location (OL) memory tests in rats exposed to ketamine alone, or combined with midazolam or dexmedetomidine. Schematic representation of the experimental design: 2A: habituation (5 days); 2B: OR1 - sample phase of OR; OR2 - choice phase, delay $15 \mathrm{~min}$, graphs represent duration of object exploration and discrimination index for object exploration in OR2; 2C: OR3 - choice phase, delay $24 \mathrm{~h}$, graphs represent duration of object exploration and discrimination index for object exploration in OR3; 2D: OL1 - sample phase of OL; OL2 - choice phase, delay $15 \mathrm{~min}$, graphs represent duration of object exploration and discrimination index for object exploration in OL2. Individual values are shown for each experimental group, $n=6$. The large horizontal bars represent mean values for each group, and the vertical bars represent the SEM. Groups: saline solution (CONTROL); $100 \mathrm{mg} / \mathrm{kg} \mathrm{ketamine} \mathrm{(KET);} 100 \mathrm{mg} / \mathrm{kg}$ ketamine and $5 \mathrm{mg} / \mathrm{kg}$ midazolam (KET/MID); and $100 \mathrm{mg} / \mathrm{kg}$ ketamine and $0.25 \mathrm{mg} / \mathrm{kg}$ dexmedetomidine (KET/DEX). No differences between groups were observed. ${ }^{*} P<0.05$, ${ }^{* *} P<0.01$ and ${ }^{* * *} P<0.001$ indicate significant differences within groups.

$(57.2 \quad[-13.9-128.2] \quad \mathrm{s}) \quad(U=5.000, \quad Z=-2.802$, $P<0.05)$. There were no significant differences between groups in the other studied parameters (Table 2).

In the EPM task there were no significant differences between groups (Table 3 ).

\section{Discussion}

This study shows that ketamine alone or in combination with midazolam or dexmedetomidine does not affect spatial and recognition memory or emotional reactivity in rats. We chose to perform the tests $48 \mathrm{~h}$ after anaesthesia, since we intended to study not the direct effect of the substances, but the possible longterm and side-effects when the anaesthetics were no longer in the animals. This time frame was chosen since the half-life of ketamine administered intramuscularly is $33 \mathrm{~min},{ }^{25}$ and the half-life of midazolam administered intravenously is $29.4 \mathrm{~min}^{26}$ in rats. Dexmedetomidine has been described with a half-life of approximately $2 \mathrm{~h}^{27}$

Results from anaesthesia variables revealed that animals anaesthetized with ketamine/dexmedetomidine had a lower heart rate and a greater depth of 
Table 2. Descriptive analysis, median, confidence interval $(\mathrm{Cl})$ and $P$ value for the parameters evaluated in the new object exploration (NOE) test.

\begin{tabular}{lccccc}
\hline Parameters measured & CONTROL & KET & KET/MID & KET/DEX & $P$ value \\
\hline Arena exploration (s) & $278.5(244.1-312.9)$ & $314.8(272.1-357.6)$ & $278.2(154.4-401.9)$ & $233.8(158.0-309.6)$ & 0.107 \\
Centre exploration (s) & $67.2(44.6-89.7)$ & $89.7(64.2-115.2)$ & $67.5(33.8-101.2)$ & $60.0(9.0-111.0)$ & 0.264 \\
Periphery exploration (s) & $211.3(192.8-229.8)$ & $255.2(168.7-281.7)$ & $210.7(118.3-303.1)$ & $173.2(142.1-204.3)$ & 0.264 \\
Time in the centre (\%) & $22.7(15.2-30.1)$ & $29.7(19.1-40.3)$ & $21.2(9.9-32.5)$ & $16.7(5.0-28.3)$ & 0.183 \\
Number visits centre & $67.2(48.2-86.1)$ & $93.5(57.2-129.7)$ & $71.2(36.1-106.3)$ & $47.3(13.5-81.2)$ & 0.153 \\
Corner behaviour (s) & $* 57.2(0-128.2)$ & $\# 33.7(9.0-58.4)$ & $138.7(-41.3-318.5)$ & $* \# 173.0(49.7-297.3)$ & $* 0.037$ \\
& & & & $30.0(4.73-55.3)$ & $39.7(33.7-45.6)$ \\
Latency to explore object (s) & $14.8(-3.1-32.8)$ & $19.8(4.2-35.5)$ & 0.103 \\
Object exploration (s) & $45.3(24.1-66.6)$ & $55.8(25.4-86.2)$ & $43.8(7.5-26.5)$ & $30.5(3.48-15.2)$ & 0.551 \\
Object approach (f) & $16.3(11.5-21.2)$ & $20.3(11.4-29.3)$ & $17.0(7.5-26.5)$ & $9.3(3.5-15.2)$ & 0.091 \\
Risk assessment posture (f) & $0.7(-0.2-1.5)$ & $2.2(-0.4-4.8)$ & $2.3(-1.7-6.4)$ & $0.5(-0.07-1.07)$ & 0.293 \\
Rearing (f) & $65.5(53.5-77.5)$ & $89.0(38.9-99.1)$ & $44.0(24.9-63.1)$ & $47.5(-33.4-61.6)$ & 0.072 \\
Grooming (s) & $26.8(15.7-38.0)$ & $23.0(10.7-35.3)$ & $22.8(0.2-45.4)$ & $41.2(11.0-71.4)$ & 0.346 \\
Grooming (f) & $6.2(4.8-7.6)$ & $5.2(3.2-7.1)$ & $4.2(0.5-7.8)$ & $7.7(2.3-13.1)$ & 0.329 \\
\hline
\end{tabular}

KET: $100 \mathrm{mg} / \mathrm{kg}$ of ketamine; KET/MID: $100 \mathrm{mg} / \mathrm{kg}$ of ketamine and $5 \mathrm{mg} / \mathrm{kg}$ of midazolam; KET/DEX: $100 \mathrm{mg} / \mathrm{kg}$ of ketamine and $0.25 \mathrm{mg} /$ $\mathrm{kg}$ of dexmedetomidine; CONTROL: saline injection; $n=6$; s: duration of the behaviour; f: frequency of the behaviour. Data were analysed with Kruskal-Wallis test followed by the Mann-Whitney U-test and expressed by median and $95 \% \mathrm{Cl}$. ${ }^{*} P<0.05$ indicates significant differences between control and KET/DEX; \#p $<0.05$ indicates significant difference between KET and KET/DEX.

Table 3. Parameters measured in the elevated plus maze test.

\begin{tabular}{lcccccc}
\hline Parameters measured & CONTROL & KET & KET/MID & KET/DEX & KET/DEX+A & $P$ value \\
\hline Time in open arm (\%) & $2(3-37)$ & $17(3-37)$ & $7(2-11)$ & $6(2-9)$ & $18(5-31)$ & 0.267 \\
Time in close arm (\%) & $63(47-79)$ & $68(42-93)$ & $76(62-91)$ & $82(77-88)$ & $67(50-83)$ & 0.357 \\
Time in centre (\%) & $17(11-23)$ & $15(7-23)$ & $16(4-30)$ & $12(7-17)$ & $15(8-22)$ & 0.935 \\
Open arm entries (f) & $3.5(1.9-5.1)$ & $2.7(0.1-5.3)$ & $2.0(0.6-3.4)$ & $2.0(1.2-2.8)$ & $3.4(0.8-6.1)$ & 0.520 \\
Close arm entries (f) & $5.8(4.2-7.5)$ & $4.0(3.2-4.6)$ & $5.0(2.3-7.7)$ & $3.1(2.0-4.3)$ & $2.1(2.1-6.5)$ & 0.520 \\
Total arm entries (f) & $9.3(7.3-11.4)$ & $6.7(3.6-9.8)$ & $7.0(3.6-10.4)$ & $5.1(4.2-6.1)$ & $7.7(3.3-12.2)$ & 0.261 \\
Locomotion (s) & $21.2(3.8-38.5)$ & $9.1(4.6-13.7)$ & $10.9(5.6-16.3)$ & $6.8(2.2-11.4)$ & $10.3(3.1-17.5)$ & 0.524 \\
Head dipping (f) & $11.7(3.1-20.3)$ & $10.7(3.0-18.4)$ & $8.0(2.7-13.3)$ & $5.4(3.1-7.8)$ & $9.6(4.1-15.0)$ & 0.432 \\
Rearing (s) & $37.9(-16.6-59.1)$ & $38.1(14.6-61.6)$ & $28.8(16.9-40.7)$ & $23.7(13.8-67.1)$ & $40.5(17.4-30.2)$ & 0.508 \\
Immobility (s) & $21.7(-10.3-53.8)$ & $53.2(-7.0-77.5)$ & $50.9(-15.2117)$ & $74.3(37.2-111)$ & $43.7(-9.6-97.0)$ & 0.294 \\
SAP (f) & $8(2.9-13.1)$ & $5.3(0.6-9.9)$ & $5.4(-0.2-11.1)$ & $4.9(0.8-8.8)$ & $4.3(1.5-7.1)$ & 0.694 \\
Latency (s) & $63.5(13.2-113.8)$ & $128.3(2.1-254.5)$ & $123(5.9-251.9)$ & $75.1(-7.1-157.4)$ & $64.9(-33.2-162.9)$ & 0.948 \\
Grooming (s) & $2.9(-4.5-10.2)$ & $4.7(-2.7-12.2)$ & $4.4(-2.9-11.8)$ & $8.5(-2.4-19.4)$ & $7.2(-4.9-19.3)$ & 0.592 \\
No. feacal boli (f) & $2.7(0.9-4.4)$ & $3.3(0.6-5.9)$ & $3.1(0.9-5.4)$ & $2.1(0.3-3.8)$ & $3.1(0.6-5.7)$ & 0.875 \\
\hline
\end{tabular}

$\mathrm{KET}: 100 \mathrm{mg} / \mathrm{kg}$ of ketamine; KET/MID: $100 \mathrm{mg} / \mathrm{kg}$ of ketamine and $5 \mathrm{mg} / \mathrm{kg}$ of midazolam; KET/DEX: $100 \mathrm{mg} / \mathrm{kg}$ of $\mathrm{ketamine}$ and $0.25 \mathrm{mg} /$ $\mathrm{kg}$ of dexmedetomidine; KET/DEX+A: $100 \mathrm{mg} / \mathrm{kg}$ of ketamine and $0.25 \mathrm{mg} / \mathrm{kg}$ of dexmedetomidine reverted with $5 \mathrm{mg} / \mathrm{kg}$ of atipamezole one hour post-anaesthesia; CONTROL: saline injection; SAP: Stretched Attend Posture; $n=7$, except for control with 6 ; f: frequency of the behaviour. Data are reported as median and $95 \%$ confidence interval.

anaesthesia when compared with ketamine / midazolam treated rats. ${ }^{28}$ Indeed, the pedal withdrawal reflex was only lost in rats from the KET/DEX and KET/ $\mathrm{DEX}+\mathrm{A}$ groups, implying that surgical anaesthesia was only achieved with these combinations. Nevertheless, the other protocols have been proven to be useful in other situations. For example, ketamine alone causes a loss of righting reflex and induces a shallow plane of anaesthesia for $30 \mathrm{~min}$, which is suitable for imaging procedures when only immobility is required. In addition to imaging, ketamine/midazolam anaesthesia may also be adequate for minor procedures such as blood collection, venepuncture/catheter placement, and subcutaneous implantation of devices.

The heart rate of animals treated with KET was higher than that of the KET/DEX and KET/DEX+A rats. In general, these results are in agreement with those found in the literature. Ketamine is expected to 
oppose the depressant actions of $\alpha_{2}$-agonists and benzodiazepines on the circulatory system. ${ }^{16,29,30}$ In humans, the combination of ketamine with dexmedetomidine provides a stable anaesthesia, with a reduction of adverse effects caused by ketamine, such as postanaesthetic delirium. ${ }^{16}$ Furthermore, the use of $\alpha_{2}$ agonists reduces anaesthetic requirements and improves perioperative haemodynamic stability. ${ }^{31,32}$ In addition, ketamine/dexmedetomidine anaesthesia has the advantage of allowing rapid recovery with the administration of atipamezole, a specific $\alpha_{2}$ antagonist available for animal use, which immediately reverses dexmedetomidine effects. ${ }^{33}$ Both animal and human studies have shown that ketamine combined with midazolam and diazepam results in fewer side-effects and better recovery than the use of ketamine alone. ${ }^{34,35}$ However, there is still a lack of information about potential secondary effects on cognitive functions of these combined drugs.

Although the scope of this study was to evaluate the effects of ketamine combinations, the use of $100 \mathrm{mg} / \mathrm{kg}$ of ketamine alone was important as a control dose for the anaesthetic combinations used. As has already been mentioned, several studies have reported ketamineinduced neurotoxicity and psychotic symptoms. Therefore, if anaesthetic combinations result in memory impairment or changes in anxiety levels, it is imperative to rule out the possibility of a direct influence of ketamine alone. In this sense, the results of animals treated with ketamine alone may provide insight for other researchers.

The behavioural analysis performed in this study demonstrated that spatial and recognition memory were unimpaired in adult rats $48 \mathrm{~h}$ post-anaesthesia with ketamine alone or in combination with midazolam or dexmedetomidine. All groups learned the radial maze task, as shown by the decreasing number of working and reference memory errors over time. Furthermore, all animals showed a decrease in response latency, a measure that seems to reflect appetitive motivational demands. ${ }^{36}$ Thus, it is suggested that the anaesthetic protocols used did not induce alterations in the motivation to locate the rewards, which is essential for a proper performance of the complex learning tasks used in neuroscience research.

Data concerning the effects of the combined administration of ketamine with midazolam or dexmedetomidine on spatial memory in rodents are scarce. Nevertheless, a previous study using the T-maze test showed that ketamine alone or in combination with medetomidine had no effects on spatial learning in mice, ${ }^{20}$ and also that ketamine alone did not interfere with radial maze learning. ${ }^{24}$ Similar results were obtained in mice treated with ketamine/midazolam when tested in the radial maze. ${ }^{18}$ Nevertheless, sub-anaesthetic ${ }^{37}$ and anaesthetic ${ }^{38}$ doses of ketamine, administered minutes before the spatial tasks caused impairment in the acquisition of the referred tasks. With the same anaesthetic dose $(100 \mathrm{mg} / \mathrm{kg}$ ), our results shows that ketamine alone has no effect on spatial memory, suggesting that an increased time period between administration and training could be enough to avoid these ketamine-induced effects on memory acquisition.

In the present study, animals from the different experimental groups exhibited a robust recognition memory, as defined by the preferential exploration of the novel object/location over the familiar object/location, and a similar discrimination index. In the test phase of the OR trial, animals have to detect and to encode a novel object, while making the update and reconsolidation of the familiar object. ${ }^{39}$ Even in the more demanding trials, with a long delay $(24 \mathrm{~h})$ between the sample and the choice phase, this cognitive process was not impaired using our anaesthesia protocols. Corroborating our results, Pitsikas and colleagues ${ }^{40}$ have reported that acute administration of ketamine $(100 \mathrm{mg} / \mathrm{kg})$ did not induce impairments in recognition memory, $48 \mathrm{~h}$ or $72 \mathrm{~h}$ after treatment and with a $24 \mathrm{~h}$ delay for object recognition or object location, respectively. Hou et $\mathrm{al}^{41}$ have also reported that the same ketamine dose did not interfere with the retention of the novel object recognition memory in mice. Furthermore, this anaesthetic procedure had no effect, up to seven days post-treatment, ${ }^{41}$ on neurons of the prefrontal cortex and hippocampus, which are the brain regions responsible for recognition, spatial memory, ${ }^{42}$ and working memory. ${ }^{43}$ It has been previously demonstrated by our group that chronic lower doses of ketamine $(5 \mathrm{mg} / \mathrm{kg})$ impaired performance in the OR/L test. ${ }^{13}$ To our knowledge, the present work is the first study to assess how a single dose of both combinations of ketamine/midazolam and ketamine/dexmedetomidine affects object recognition memory.

In the NOE test, animal responses may reflect anxiety and/or fear, which is reflected in the avoidance activity or exploring the new object. ${ }^{44}$ Here, all groups exhibited a similar response to the new object; in general, all treatment groups had emotional reactivity similar to control levels $48 \mathrm{~h}$ post-anaesthesia. However, rats in the KET/DEX group spent significantly more time in corner behaviour than the CONTROL and KET groups. This behaviour represents a thigmotactic response, a natural defensive response in which rats remain close to vertical surfaces. Therefore, this result suggests that ketamine/dexmedetomidine treated animals show an increased anxiety and/ or fear-like behaviour.

In order to corroborate the NOE results, a more robust and classical test for anxiety, EPM, was performed. The space occupation in this task reflects a 
conflict between the rodent's preference for protected areas (e.g. closed arms) and their innate motivation to explore novel environments, such as the open arms. ${ }^{45}$ An increased time spent and a higher number of entries in the open arms are generally interpreted as a decrease in anxiety-like behaviour. ${ }^{46}$ Since the differences detected in the NOE test involved the group (KET/ DEX) that had anaesthesia duration more than three times longer than the other groups, we also tested the KET/DEX+A group. These animals were treated with ketamine/dexmedetomidine and, after one hour, anaesthesia was reverted with atipamezole (an $\alpha 2$-adrenergic antagonist). This procedure was included in order to control anaesthesia duration, which could be a variable inducing the results previously referred to. Our data showed that the different anaesthetic protocols used did not affect space occupation in the EPM apparatus. Other ethological measures registered, such as the number of rears, head dips, faecal boli, freezing or stretched-attend postures, were also not affected by the anaesthetic protocols.

Overall, our results clarified the NOE test outcomes, supporting the absence of effects of the anaesthetic protocols used on emotional behaviour. Low ketamine doses, such as $20 \mathrm{mg} / \mathrm{kg}$, may be anxiogenic, ${ }^{47}$ while doses which are higher, but lower than our dose, result in anxiolytic-related behaviour. ${ }^{48}$ Nevertheless, these results were obtained when ketamine was administered a few minutes to $2 \mathrm{~h}$ before the EPM test. In humans, studies of anxiety induced by ketamine are few; and in these studies ketamine does not seem to interfere with anxiety issues, even three days after administration. ${ }^{49}$ These studies are in agreement and support, to some extent, our data obtained $48 \mathrm{~h}$ postanaesthesia. Concerning ketamine combinations, little is known about their effects on anxiety, except that dexmedetomidine and midazolam are considered to be anxiolytics. ${ }^{16,30}$ In humans, both drugs may be given to ketamine-anaesthetized patients in order to induce sedation and anxiolysis; however, these effects are not usually evaluated in the long term, such as after discharge from the post-anaesthesia care unit. ${ }^{16}$

Therefore, besides the described ketamine sideeffects, ${ }^{6}$ it is important to highlight the absence of ketamine memory impairment and alterations on emotional reactivity in our results. These outcomes contradict several reports in rodents which can be explained by methodological differences such as different dose effects (sub-anaesthetic versus anaesthestic $\operatorname{doses}^{37}$ ), age (young and elderly may be more susceptible to anaesthetic toxicity ${ }^{50,51}$ ), frequency of administration (acute versus chronic ${ }^{13}$ ), time between anaesthesia and behavioural evaluation, ${ }^{38}$ study of different memory processes, ${ }^{52}$ and temperature. ${ }^{40}$ In accordance, human studies have consistently reported that acute ketamine administration induces short-term psychotic events and transient memory impairment without causing sequelae. ${ }^{53}$ With these reports, the variables inherent in clinical settings, painful and stressful procedures, and the large diversity of individuals must all be taken into consideration. The use of animal models enables us to overcome these limitations by using a controlled environment, and no surgery or other invasive procedures in order to study the sole effects of anaesthetics.

As previously stated, the potential effects of anaesthesia in neurobehavioural experiments depend on several factors; thus, there is not simply one appropriate time interval from anaesthesia to behavioural assessments to avoid anaesthesia-related effects. Nevertheless, neurobehavioural experiments may be conducted without expecting long-term anaesthesia effects $48 \mathrm{~h}$ after a single injection of $100 \mathrm{mg} / \mathrm{kg}$ ketamine and its combinations (midazolam, medetomidine, dexmedetomidine). ${ }^{18,19,20,24}$ Other anaesthetic protocols may be used for this purpose, such as high concentrations of isoflurane, ${ }^{54,55}$ sevoflurane and desflurane. ${ }^{56}$ However, injectable anaesthesia has the advantage of not requiring any special equipment as volatile anaesthesia does. In addition, injectable anaesthetics are needed to promote balanced anaesthesia. Other anaesthetic protocols such as propofol/medetomidine/fentanyl, ${ }^{57,58}$ and ketamine combined with other benzodiazepines (diazepam) and $\alpha-2$ agonists (xylazine) are suitable for use in laboratory rodents $;{ }^{59}$ however, further studies are needed regarding their effects at the neurobehavioural level.

In summary, our results show that despite the unconsciousness time varying across the anaesthetic protocols used (ketamine alone, or in combination with midazolam or dexmedetomidine), spatial, recognition memory and anxiety-like behaviours are not affected in adult rats. These results indicate that, in adult rats, the tested anaesthetic combinations and doses do not induce long-term effects regarding memory. Although midazolam and dexmedetomidine are considered to have anxiolytic properties, our results show that after $48 \mathrm{~h}$, these drugs do not affect emotional behaviour. Thus, our results suggest that these combinations are useful for rat anaesthesia and/or sedation procedures in clinical and neurobehavioural research without compromising the quality of the outcomes. Therefore, the use of anaesthesia in laboratory rodents, namely these protocols, should always be considered in order to refine procedures and decrease rodent pain and distress.

\section{Acknowledgements}

A Magalhães was responsible for study design, data collection, data analysis and drafting of the article; A. Valentim was responsible for study design, data collection, data analysis and article revision; M Pereira was responsible for data analysis; P Melo was responsible for data collection and 
article revision; C Venâncio was responsible for study design, data collection and article revision; $\mathrm{T}$ Summavielle was responsible for study design and article revision; L Antunes was responsible for study design, article revision and approval of the final version to be published.

\section{Declaration of Conflicting Interests}

The author(s) declared no potential conflicts of interest with respect to the research, authorship, and/or publication of this article.

\section{Funding}

The author(s) disclosed receipt of the following financial support for the research, authorship, and/or publication of this article: This research project was financed by Fundação para a Ciência e Tecnologia (FCT) through the research grant PTDC/CVT/099022/2008 and financed by COMPETE: -010124- FEDER -009497 (Lisbon, Portugal). Teresa Summavielle was supported by Programa Ciência Programa Operacional Potencial Humano (POPH)Promotion of Scientific Employment, ESF and MCTES and program Investigador FCT, POPH and Fundo Social Europeu. Ana Magalhães was supported by PTDC/MHCPAP/5304/2012. Luis Antunes and Carlos Venâncio were supported by the European Investment Funds by FEDER/ COMPETE/POCI Operacional Competitiveness and Internacionalization Programme, under Project POCI-010145-FEDER-006958 and National Funds by FCT under the project UID/AGR/04033/2013.

\section{References}

1. Cruz JI, Loste JM and Burzaco OH. Observations on the use of medetomidine/ketamine and its reversal with atipamezole for chemical restraint in the mouse. Lab Anim 1998; 32: 18-22.

2. Arnemo JM, Storaas T, Khadka CB and Wegge P. Use of medetomidine-ketamine and atipamezole for reversible immobilization of free-ranging hog deer (Axis porcinus) captured in drive nets. $J$ Wildlife Dis 2005; 41: 467-470.

3. Russell WMS and Burch R. The principles of humane experimental technique. London: Methuen, 1959.

4. Himmelseher S and Durieux ME. Revising a dogma: ketamine for patients with neurological injury? Anesth Analg 2005; 101: 524-534.

5. Rice MJ, Gwertzman A, Finley $\mathrm{T}$ and Morey TE. Anesthetic practice in Haiti after the 2010 earthquake. Anesth Analg 2010; 111: 1445-1449.

6. Morgan CJ, Mofeez A, Brandner B, Bromley L and Curran HV. Acute effects of ketamine on memory systems and psychotic symptoms in healthy volunteers. Neuropsychopharmacology 2004; 29: 208-218.

7. Slonim AD and Ognibene FP. Sedation for pediatric procedures, using ketamine and midazolam, in a primarily adult intensive care unit: a retrospective evaluation. Crit Care Med 1998; 26: 1900-1904.

8. Tobias JD, Gupta P, Naguib A and Yates AR. Dexmedetomidine: applications for the pediatric patient with congenital heart disease. Pediatr Cardiol 2011; 32: 1075-1087.

9. Pain L, Launoy A, Fouquet $\mathrm{N}$ and Oberling $\mathrm{P}$. Mechanisms of action of midazolam on expression of contextual fear in rats. Br J Anaesth 2002; 89: 614-621.

10. Arcangeli A, D'Alo C and Gaspari R. Dexmedetomidine use in general anaesthesia. Curr Drug Targets 2009; 10: 687-695.

11. Morgan CJ and Curran HV. Acute and chronic effects of ketamine upon human memory: a review. Psychopharmacology (Berl) 2006; 188: 408-424.

12. Duan TT, Tan JW, Yuan Q, Cao J, Zhou QX and Xu L. Acute ketamine induces hippocampal synaptic depression and spatial memory impairment through dopamine D1/D5 receptors. Psychopharmacology (Berl) 2013; 228: 451-461.

13. Venancio C, Magalhaes A, Antunes L and Summavielle T. Impaired spatial memory after ketamine administration in chronic low doses. Curr Neuropharmacol 2011; 9: 251-255.

14. Hudetz JA, Iqbal Z, Gandhi SD, et al. Ketamine attenuates post-operative cognitive dysfunction after cardiac surgery. Acta Anaesth Scand 2009; 53: 864-872.

15. Wagner BK, O'Hara DA and Hammond JS. Drugs for amnesia in the ICU. Am J Crit Care 1997; 6: 192-201. (quiz 202-203).

16. Levanen J, Makela $\mathrm{ML}$ and Scheinin $\mathrm{H}$. Dexmedetomidine premedication attenuates ketamineinduced cardiostimulatory effects and postanesthetic delirium. Anesthesiology 1995; 82: 1117-1125.

17. Saint-Maurice C, Landais A, Delleur MM, Esteve C, MacGee K and Murat I. The use of midazolam in diagnostic and short surgical procedures in children. Acta Anaesthesiol Scand Suppl 1990; 92: 39-41. (discussion 47).

18. Valentim AM, Olsson IA and Antunes LM. The anaesthetic combination of ketamine/midazolam does not alter the acquisition of spatial and motor tasks in adult mice. Lab Anim 2013; 47: 19-25.

19. Valentim AM, Ribeiro PO, Olsson IA and Antunes LM. The memory stages of a spatial Y-maze task are not affected by a low dose of ketamine/midazolam. Eur $J$ Pharmacol 2013; 712: 39-47.

20. Ribeiro PO, Valentim AM, Rodrigues P, Olsson IA and Antunes LM. Apoptotic neurodegeneration and spatial memory are not affected by sedative and anaesthetics doses of ketamine/medetomidine combinations in adult mice. Br J Anaesth 2012; 108: 807-814.

21. Dudchenko PA. An overview of the tasks used to test working memory in rodents. Neurosci Biobehav Rev 2004; 28: 699-709.

22. Ennaceur A, Michalikova S, Bradford A and Ahmed S. Detailed analysis of the behavior of Lister and Wistar rats in anxiety, object recognition and object location tasks. Behav Brain Res 2005; 159: 247-266.

23. Culley DJ, Baxter MG, Yukhananov R and Crosby G. Long-term impairment of acquisition of a spatial memory task following isoflurane-nitrous oxide anesthesia in rats. Anesthesiology 2004; 100: 309-314.

24. Ribeiro PO, Rodrigues PC, Valentim AM and Antunes LM. A single intraperitoneal injection of ketamine does not affect spatial working, reference memory or 
neurodegeneration in adult mice: an animal study. Eur $J$ Anaesth 2013; 30: 618-626.

25. Williams ML, Mager DE, Parenteau H, et al. Effects of protein calorie malnutrition on the pharmacokinetics of ketamine in rats. Drug Metab Dispos 2004; 32: 786-793.

26. Kotegawa T, Laurijssens BE, Von Moltke LL, et al. In vitro, pharmacokinetic, and pharmacodynamic interactions of ketoconazole and midazolam in the rat. J Pharmacol Exp Ther 2002; 302: 1228-1237.

27. EMA (European Medicine Agency). Dexdomitor: EPAR - scientific discussion. European public assessment report, http://www.ema.europa.eu/docs/en_GB/document_library/EPAR_-_Scientific_Discussion/veterinary/ 000070/WC500062496.pdf (2002, accessed 18 December 2015).

28. Kenny GN and Mantzaridis H. Closed-loop control of propofol anaesthesia. Br J Anaesth 1999; 83: 223-228.

29. Reich DL and Silvay G. Ketamine: an update on the first twenty-five years of clinical experience. Can J Anaesth 1989; 36: 186-197.

30. Jorden VSB and Tung A. Dexmedetomidine: clinical update. Sem Anesth Periop Med Pain 2002; 21: 265-274.

31. Hall JE, Uhrich TD, Barney JA, Arain SR and Ebert TJ. Sedative, amnestic, and analgesic properties of small-dose dexmedetomidine infusions. Anesth Analg 2000; 90: 699-705.

32. Tanaka M and Nishikawa T. Oral clonidine premedication attenuates the hypertensive response to ketamine. $\mathrm{Br}$ J Anaesth 1994; 73: 758-762.

33. Scheinin H, Aantaa R, Anttila M, Hakola P, Helminen A and Karhuvaara S. Reversal of the sedative and sympatholytic effects of dexmedetomidine with a specific $\alpha 2$-adrenoceptor antagonist atipamezole: a pharmacodynamic and kinetic study in healthy volunteers. Anesthesiology 1998; 89: 574-584.

34. Flecknell PA. Laboratory animal anaesthesia: a practical introduction for research workers and technicians. London: Academic Press, 1996.

35. Toft $\mathrm{P}$ and Romer U. Comparison of midazolam and diazepam to supplement total intravenous anaesthesia with ketamine for endoscopy. Can J Anaesth 1987; 34: 466-469.

36. Ormerod BK and Beninger RJ. Water maze versus radial maze: differential performance of rats in a spatial delayed match-to-position task and response to scopolamine. Behav Brain Res 2002; 128: 139-152.

37. Chrobak JJ, Hinman JR and Sabolek HR. Revealing past memories: proactive interference and ketamine-induced memory deficits. J Neurosci 2008; 28: 4512-4520.

38. Moosavi M, Khales GY, Rastegar K and Zarifkar A. The effect of sub-anesthetic and anesthetic ketamine on water maze memory acquisition, consolidation and retrieval. Eur J Pharmacol 2012; 677: 107-110.

39. Ennaceur A. One-trial object recognition in rats and mice: methodological and theoretical issues. Behav Brain Res 2010; 215: 244-254.

40. Pitsikas N and Boultadakis A. Pre-training administration of anesthetic ketamine differentially affects rats' spatial and non-spatial recognition memory. Neuropharmacology 2009; 57: 1-7.
41. Hou Y, Zhang H, Xie G, et al. Neuronal injury, but not microglia activation, is associated with ketamine-induced experimental schizophrenic model in mice. Prog Neuropsychopharmacol Biol Psychiatry 2013; 45: 107-116.

42. Broadbent NJ, Squire LR and Clark RE. Spatial memory recognition |memory, and the hippocampus. Proc Natl Acad Sci USA 2004; 101: 14515-14520.

43. Curtis CE and D'Esposito M. Persistent activity in the prefrontal cortex during working memory. Trends Cogn Sci 2003; 7: 415-423.

44. Hughes RN. Intrinsic exploration in animals: motives and measurement. Beha Processes 1997; 41: 213-226.

45. Walf AA and Frye CA. The use of the elevated plus maze as an assay of anxiety-related behavior in rodents. Nat Protocols 2007; 2: 322-328.

46. Rodgers RJ, Haller J, Holmes A, Halasz J, Walton TJ and Brain PF. Corticosterone response to the plus-maze: high correlation with risk assessment in rats and mice. Physiol Behav 1999; 68: 47-53.

47. da Silva FCC, do Carmo de Oliveira Cito M, da Silva MIG, et al. Behavioral alterations and pro-oxidant effect of a single ketamine administration to mice. Brain Res Bull 2010; 83: 9-15.

48. Engin E, Treit D and Dickson CT. Anxiolytic- and antidepressant-like properties of ketamine in behavioral and neurophysiological animal models. Neuroscience 2009; 161: 359-369.

49. Morgan CJA, Mofeez A, Brandner B, Bromley L and Curran HV. Ketamine impairs response inhibition and is positively reinforcing in healthy volunteers: a doseresponse study. Psychopharmacology 2004; 172: 298-308.

50. Jevtovic-Todorovic V and Carter LB. The anesthetics nitrous oxide and ketamine are more neurotoxic to old than to young rat brain. Neurobiol Aging 2005; 26: 947-956.

51. Young C, Jevtovic-Todorovic V, Qin YQ, et al. Potential of ketamine and midazolam, individually or in combination, to induce apoptotic neurodegeneration in the infant mouse brain. Br J Pharmacol 2005; 146: 189-197.

52. Boultadakis A and Pitsikas N. Anesthetic ketamine impairs rats' recall of previous information: the nitric oxide synthase inhibitor N-nitro-L-arginine methylester antagonizes this ketamine-induced recognition memory deficit. Anesthesiology 2011; 114: 1345-1353.

53. Perry EB, Cramer JA, Cho HS, et al. Psychiatric safety of ketamine in psychopharmacology research. Psychopharmacology 2007; 192: 253-260.

54. Valentim AM, Alves HC, Olsson IA and Antunes LM. The effects of depth of isoflurane anesthesia on the performance of mice in a simple spatial learning task. $J \mathrm{Am}$ Assoc Lab Anim Sci 2008; 47: 16-19.

55. Valentim AM, Di Giminiani P, Ribeiro PO, Rodrigues P, Olsson IA and Antunes LM. Lower isoflurane concentration affects spatial learning and neurodegeneration in adult mice compared with higher concentrations. Anesthesiology 2010; 113: 1099-1108.

56. Kilicaslan A, Belviranli M, Okudan N and Nurullahoglu Atalik E. Single and repeated sevoflurane or desflurane exposure does not impair spatial memory performance of young adult mice. Fundam Clin Pharmacol 2013; 27: 641-649. 
57. Alves HC, Valentim AM, Olsson IA and Antunes LM. Intraperitoneal anaesthesia with propofol, medetomidine and fentanyl in mice. Lab Anim 2009; 43: 27-33.

58. Alves HN, da Silva AL, Olsson IA, Orden JM and Antunes LM. Anesthesia with intraperitoneal propofol, medetomidine, and fentanyl in rats. J Am Assoc Lab Anim Sci 2010; 49: 454-459.

59. Fish R, Danneman PJ, Brown $\mathrm{M}$ and Karas A. Anesthesia and analgesia in laboratory animals. New York: Academic Press, 2011. 